PATIENT SAFETY

\begin{tabular}{l|l} 
Y. Niu & BACKGROUND AND PURPOSE: Temporal bone CT is performed frequently in clinical practice. At the \\
Z. Wang & same time, the eye lens are exposed to ionizing radiation without any useful diagnostic information \\
Y. Liu & delivered. Our aim was to investigate the radiation dose to the lens of the eye by using temporal bone \\
Z & CT scanning with different protocols.
\end{tabular}

V. Yao

MATERIALS AND METHODS: Direct axial and coronal CT by using a conventional sequential scanning

\section{Radiation Dose to the Lens Using Different Temporal Bone CT Scanning Protocols} mode $(140 \mathrm{kV}, 220 \mathrm{mAs} / \mathrm{section}, 1.25-\mathrm{mm}$ thickness, $1.25-\mathrm{mm}$ increment, $16 \times 0.625 \mathrm{~mm}$ collimation, the glabellomeatal line as a scanning baseline), a routine helical scanning mode (140 kV, $220 \mathrm{mAs} /$ section, 0.315 pitch, $0.67-\mathrm{mm}$ thickness, $0.33-\mathrm{mm}$ increment, $16 \times 0.625$ collimation, the orbitomeatal line as a scanning baseline), and a modified helical scanning mode (acanthiomeatal line as a scanning baseline; other parameters, same as above) was performed on an exsomatized cadaveric head. CTDI vol $_{\text {I }}$ and DLP were recorded for each scanning mode, and effective doses were calculated. Organ doses for the lens were measured with TLDs.

RESULTS: When the sequential scanning mode was used, the gross effective dose was $1.21 \mathrm{mSv}$ and the organ dose to the lens was 50.96 and $1.73 \mathrm{mGy}$, respectively, for direct axial and coronal imaging. The effective dose was $0.803 \mathrm{mSv}$ in routine helical scanning, while the lens dose was $40.17 \mathrm{mGy}$. With the modified helical scanning mode, the effective dose was as same as that for the routine helical scanning, but the lens dose was reduced significantly to $10.33 \mathrm{mGy}$.

ConcLusions: The effective doses resulting from sequential axial and coronal scanning were 1.51 times higher than the dose from helical scanning, and the lens dose was 1.31 times higher. With the modified helical scanning mode, thinner section images could be used to reformat axial, coronal, and sagittal images with a further $74.3 \%$ reduction in lens dose beyond that achieved with the conventional helical protocol.

ABBREVIATIONS: CTDI $_{\text {vol }}=$ volume CT dose index; DLP = dose-length product; $M S C T=$ multisection $C T$; TLD $=$ thermoluminescence dosimeter.
$\mathbf{H}_{3}$ igh-resolution CT is the technique of first choice for imaging the temporal bone and enabling accurate delineation of normal temporal bone anatomy and complex osseous pathologies. When performed by using conventional sequential direct axial and coronal methods, parallel to the glabellomeatal or orbitomeatal line, the patient's eyes are positioned within the scanning range where they are exposed to radiation directly. This is a major concern, because the lens is one of the most radiation-sensitive organs in the human bodyespecially in children, in whom the risk of developing lens opacities (eg, cataracts) may be higher when considering cumulative lifetime doses. With the innovation of multisection CT technologies and postprocessing software, images parallel to the proper baseline can be obtained by reformatting thinsection helical acquisitions to axial and coronal images. In this study, we investigated the radiation dose to the lens by using temporal bone CT scanning with sequential scanning techniques and multisection helical techniques. Our goal was a lens organ dose as low as reasonably achievable while still delivering sufficient diagnostic information.

Received May 13, 2009; accepted after revision July 1

From the Department of Radiology (Y.N., Z.W., Y.L., Z.L.), Beijing Tongren Hospital, Capital Medical University, Beijing, China; and Philips Healthcare (V.Y.), Beijing, China.

Please address correspondence to Zhenchang Wang, MD, Department of Radiology, Beijing Tongren Hospital, Capital Medical University, No. 1 Dongjiaominxiang, Dongcheng District, Beijing 100730, China; e-mail: ytniu163@163.com

DOl 10.3174/ajnr.A1807

\section{Materials and Methods}

\section{Subjects}

Four exsomatized cadaveric heads were included in the study. The cadaveric heads were removed from the formalin solution and positioned upright approximately 12 hours before scanning to discharge the solution from the nasal cavity and external auditory canal. A pilot study of 4 cadaveric heads was performed to select the head most fit for the experiment.

\section{CT Technique}

All the images in this study were acquired on a Brilliance 64-section CT scanner (Philips Healthcare, Cleveland, Ohio) by using 3 scanning modes: sequential, conventional helical, and modified helical.

For the sequential scanning mode, so-called conventional direct axial and direct coronal CT was performed with $140 \mathrm{kVp}, 220 \mathrm{mAs} /$ section, and $16 \times 0.625 \mathrm{~mm}$ collimation. Images were reconstructed with a $1.25-\mathrm{mm}$ section thickness and a $1.25-\mathrm{mm}$ section interval. The axial images were acquired with the subject's neck flexed and the gantry angled in the cranial direction so that the skull base (ie, the glabellomeatal line) was parallel to the scanning plane (Fig 1). The coronal images were acquired with the neck extended in the hanginghead cradle, and the gantry was angled maximally $\left(30^{\circ}\right)$ in the caudal direction to obtain images in a plane as perpendicular to the hard palate as possible. In this study, each cadaveric head was placed on the table similar to the conventional axial and coronal CT positions.

The conventional helical scanning mode used $140 \mathrm{kVp}, 220 \mathrm{mAs} /$ section, $16 \times 0.625 \mathrm{~mm}$ collimation, and 0.315 pitch. The images 


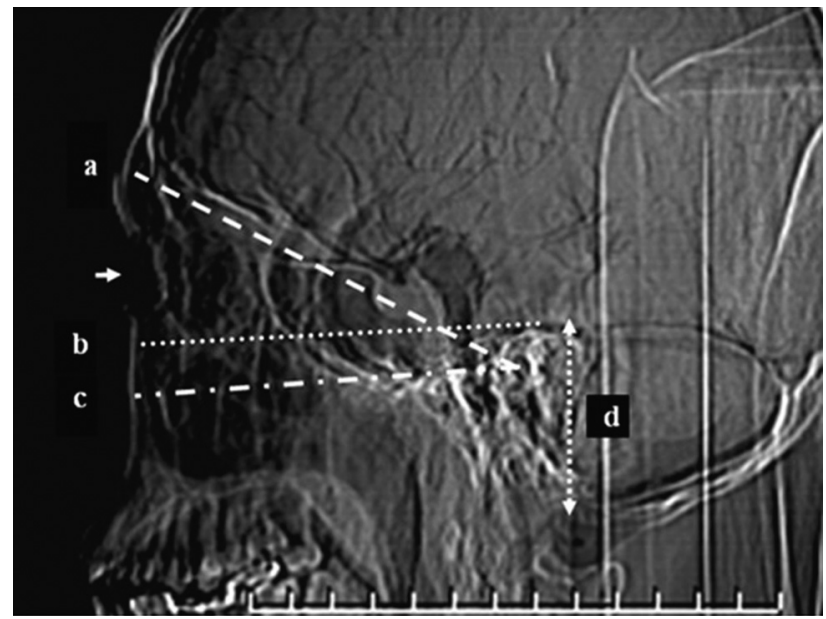

Fig 1. Scanning baseline and coverage of temporal bone CT: glabellomeatal line, the baseline of the sequential axial scanning mode (a); a line parallel to the acanthiomeatal line passing by the superior edge of the temporal bone, the upper limit of the modified helical scanning mode (b); the acanthiomeatal line approximately parallel to the hard palate (c); and the scanning range of the temporal bone CT (d). The arrow is the position of lens.

were reconstructed with a section thickness of $0.67 \mathrm{~mm}$ and an increment of $0.33 \mathrm{~mm}$. Axial, coronal, and oblique sagittal images used for clinical diagnosis were reformatted with a 1-mm section thickness and a 1-mm increment. Routine helical CT was performed without gantry angulation and with the head and neck in a neutral position (ie, the orbitomeatal line was approximately perpendicular to the table).

The scanning parameters of the modified helical scanning mode were the same as those for the conventional helical mode, with the exception of the scanning baseline. As with conventional helical scanning, no gantry angulation was used. The cadaveric head was placed on the table with the scanning baseline parallel to the acanthiomeatal line.

All scans were obtained by using a gantry rotation speed of 0.75 seconds, an 18-cm FOV, a high-resolution scanning mode, a Y-Sharp (YC, bone) filter (Philips Healthcare, Cleveland, Ohio) with an edge enhancement factor of 1 , a focal spot size of $1 \times 1 \mathrm{~mm}$, and a $512 \times$ 512 image matrix.

\section{Image Processing}

Analyses were performed by using a dedicated postprocessing workstation (Extended Brilliance Workspace, Philips Healthcare). The axial, coronal, and oblique sagittal reformatted images used for diagnosis with a section thickness of $1 \mathrm{~mm}$ and an increment of $1 \mathrm{~mm}$ were each reviewed. For each scanning mode, the clarity of the temporal bone anatomic and microanatomic structures was evaluated.

\section{Radiation Dosimetry}

A single batch of TLDs was acquired and used for this study to measure radiation dose. The TLDs were calibrated for diagnostic x-ray energies with an accuracy and precision of $10 \%$. Two TLDs were placed under the right eyelid and side-by-side at the position of the lens for each scan. The mean of the 2 TLD doses in each scan was assumed to be equal to the dose delivered to the lens in that scan.

For calculation of the effective radiation dose for the entire imaged region, DLP values were used. Estimation of the effective dose was calculated for each scan by multiplying the DLP with a standardized conversion factor of $0.0031 \mathrm{mSv} \mathrm{mGy}^{-1} \mathrm{~cm}^{-1}$ for examinations of the head and neck (including the temporal bone). ${ }^{1}$ The DLP was provided automatically by the scanner and is the product of the
$\mathrm{CTDI}_{\mathrm{vol}}$ and the length of the exposed volume (ie, the scanning range).

The effective dose, $E$, is related to the DLP as follows:

$$
E=k \times D L P(m S v),
$$

where the unit of DLP is $\mathrm{mGy} / \mathrm{cm}$ and $k$ is the region-specific normalized effective dose conversion factor $\left(\mathrm{mSv} \mathrm{mGy}^{-1} \mathrm{~cm}^{-1}\right)$.

\section{Results}

The images obtained from sequential axial and coronal scans are shown in Fig $2 A,-B$. Figures $2 C-F$ are axial, coronal, and oblique sagittal images reformatted from the modified helical scanning data. All the images delineate the microanatomy of the temporal bone clearly and can be used for clinical diagnosis.

In sequential axial and coronal scanning, it was necessary to tilt the gantry. As a result of this angulation, the corresponding CTDI $_{\mathrm{vol}}$ changed as shown in Table 1.

As can be seen in Table 2, the combined effective dose of the direct axial and coronal scans was $1.21 \mathrm{mSv}$, compared with a total effective dose of $0.803 \mathrm{mSv}$ in either the routine or modified helical scans. According to the organ-dose values obtained from the TLDs, the lens dose in the modified helical scan was $10.33 \mathrm{mGy}$, which was much lower than the 52.69 mGy of the sequential scan and the $40.17 \mathrm{mGy}$ of the routine helical scan. The lens dose in the coronal scan was $1.73 \mathrm{mGy}$, the lowest of all scans.

The lens doses for sequential axial scans with 3 different coverages of 40,60, and $80 \mathrm{~mm}$ are shown in Table 3 .

\section{Discussion}

During temporal bone CT, radiation exposure of the eye lens is unavoidable. Therefore, it is very important to reduce the radiation exposure as much as possible to reduce the deterministic effects of radiation on the lens. In this study, we compared the effective dose and lens organ dose of 3 temporal bone CT scanning modes: sequential, routine helical, and modified helical.

In our department, the baseline used for sequential temporal bone scanning is parallel to the glabellomeatal line; however, the American College of Radiology recommends that the infraorbitomeatal line be used as the baseline. ${ }^{2}$ When the infraorbitomeatal line is the baseline, the lenses are also included in the scanning range and receive direct radiation exposure. Once the lenses are included in the scanning range, the lens organ dose does not change substantially (only $0.66 \mathrm{mGy}$ ) as coverage increases from 40 to $80 \mathrm{~mm}$.

The lens dose from a cranial CT scan varies from 22.4 to $100 \mathrm{mGy}$, depending on beam width, reconstruction interval, tube voltage, tube current, angulation, coverage, etc. ${ }^{3-8}$ To reduce the lens dose, the 2 methods often used are the following: changing the scanning baseline to avoid the lens or adjusting the scanning parameters to reduce the dose without changing the baseline. From the point of view of organ dose, the former can decrease lens dose considerably, and the latter can reduce the dose to a certain extent while increasing image noise notably. Torizuka et $\mathrm{al}^{9}$ evaluated the radiation dose to the lens and the visualization of temporal bone structures by scanning along the orbitomeatal line parallel to the hard palate. The 

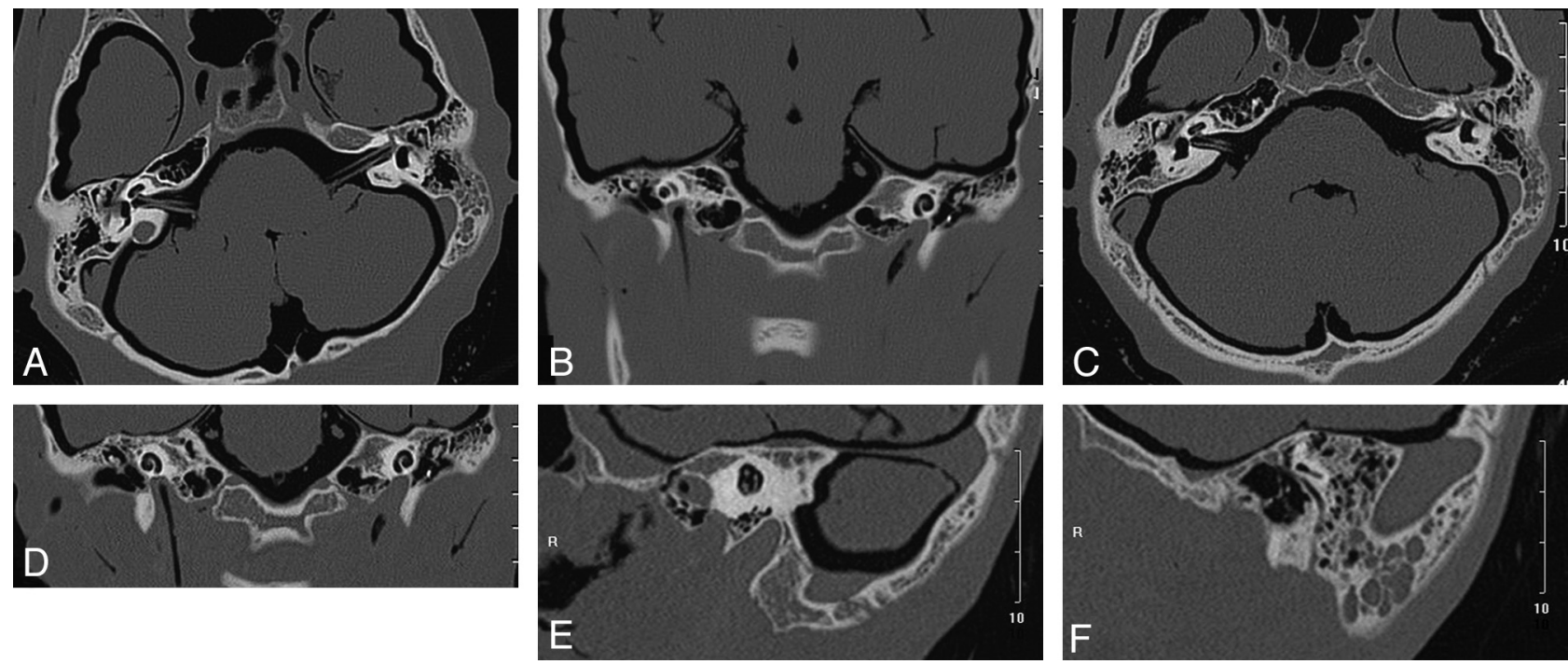

Fig 2. Sequential axial and coronal CT images and reformatted images from the modified helical scan. $A$ and $B$, Sequential axial section of the vestibulum auris ( $A$ ) and a coronal section of the cochlea $(B)$, with the structures delineated vividly. $C-F$, Axial, coronal, and sagittal reformatted sections from the modified helical scan with the microanatomy displayed clearly and more diagnostic information delivered.

\begin{tabular}{lccc}
\hline \multicolumn{4}{l}{ Table 1: CTDI $_{\text {vol }}$ as a function of gantry angulation } \\
\hline \\
\hline Tilt & CTDI $_{\text {vol }}$ (mGy) & Tilt & CTDI $_{\text {vol }}$ (mGy) \\
\hline 30 & 44.8 & 0 & 51.6 \\
25 & 46.9 & -5 & 51.6 \\
20 & 48.6 & -10 & 51.1 \\
15 & 50.1 & -15 & 50.1 \\
10 & 51.1 & -20 & 48.6 \\
5 & 51.6 & -25 & 46.9 \\
\hline
\end{tabular}

a Scanning parameters were identical for each angle. Positive tilt values correspond to the caudal direction; negative tilt values correspond to the cranial direction.

Table 2: The effective doses and lens doses in different temporal bone CT scanning protocols

\begin{tabular}{lccc}
\hline Scanning Mode & $\begin{array}{c}\text { CTDI }_{\text {vol }} \\
\text { (mGy) }\end{array}$ & $\begin{array}{c}\text { Effective Dose } \\
\text { (mSv) }\end{array}$ & $\begin{array}{c}\text { Lens Organ } \\
\text { Dose } \\
\text { (mGy) }\end{array}$ \\
\hline Sequential axial & 51.6 & $0.640,1.21$ & $50.96,52.69$ \\
Sequential coronal & 46.0 & 0.570 & 1.73 \\
Routine multisection helical & 51.6 & 0.803 & 40.17 \\
Modified multisection helical & 51.6 & 0.803 & 10.33 \\
\hline
\end{tabular}

\begin{tabular}{|c|c|}
\hline Coverage (mm) & Lens Dose (mGy) \\
\hline 40 & 50.96 \\
\hline 60 & 51.53 \\
\hline 80 & 51.62 \\
\hline
\end{tabular}

change of the baseline decreased the lens dose from 12.7 to 0.274 cGy but resulted in difficulty in evaluating the pneumatic space of the temporal bone and the auditory ossicles. However, most previous studies have focused their attention on reducing the dose through reductions in scanning parameters such as tube voltage and tube current. Funama et al ${ }^{10}$ investigated lens-dose reduction in pediatric high-resolution CT temporal bone scanning and reported satisfactory image quality after reducing the tube current to $120 \mathrm{mAs}$. Some researchers have suggested that tube current should be set between 150 and $200 \mathrm{mAs}$ to protect the lens, while others have concurred with Funama et $\mathrm{al}^{10}$ and found that satisfactory images could be acquired with as low as $120 \mathrm{mAs} .^{11,12}$ Additional studies on low-dose pediatric temporal bone CT recommended further reductions in the scanning technique to 120 $\mathrm{kV}, 50-75 \mathrm{mAs}$, and 0.75 pitch. ${ }^{13-15}$ Other authors have reported using a heavy metal (eg, bismuth) shield to enable up to $50 \%$ reduction in radiation to the lens during routine cranial CT; however, patients felt discomfort, and image artifacts in near the eye were serious. ${ }^{16}$

High-resolution thin-section direct axial and coronal CT is the standard technique for temporal bone imaging. The axial scanning baseline is usually the glabellomeatal line with mandible adduction and gantry tilt in the cranial direction. This leads to a higher lens organ dose because the lens receives direct radiation exposure. During coronal scanning, the lenses receive lower doses from scattered radiation. To compare the 2 sides of the temporal bone, absolute bilateral symmetry in both axial and coronal sections is required. In addition, the direct coronal technique requires significant neck extension (hanging-head position) and a special head holder. Consequently, repeated acquisitions are often needed after repositioning the patient's head, leading to decreased throughput and increased discomfort and radiation dose.

With the advent of and widespread adoption of MSCT, many have investigated the quality of the delineation of temporal bone anatomy and the radiation dose associated with multisection helical scanning techniques. Caldemeyer et $\mathrm{al}^{17}$ have shown that helical CT using a $0.5-\mathrm{mm}$ reconstruction produces diagnostic images comparable with or superior to conventional 1-mm reconstructions. Another study indicated that compared with 4-section CT, a low-dose 64-section CT technique facilitates temporal bone imaging with sufficient anatomic detail. ${ }^{18}$ The lens dose on a multisection scanner is still substantially less than the threshold dose of 0.5-2.0 Gy, which has been attributed to detectable lens opacities. ${ }^{19}$ However, dose reduction is still a priority, given that some patients need many CT scans. This cumulative dose is of particular concern for children and other populations who are more sensitive to radiation. 
The submillimeter collimation available with MSCT allows thin sections to be reformatted to axial, coronal, and oblique sagittal images by using advanced processing techniques. These reformatted images deliver more anatomic information to physicians and give greater flexibility in imaging. For example, patient position can be changed to have the mandible extended with the acanthiomeatal line perpendicular to the table in order to allow the lens to be moved out of the scanning range. Such a helical scan with no gantry angulation yields images parallel to the hard palate that have a different appearance compared with routine images; however, images that correspond to those resulting from conventional scanning with a baseline of the glabellomeatal line can be easily generated with flexible postprocessing tools. The operator must locate the lens $1 \mathrm{~cm}$ above the upper edge of the scanning range to avoid direct radiation because of overranging and reduced scatter radiation to the lenses. If the lens is the upper bound of the scanning range, the lens dose increases greatly. The lens dose varies little with scanning coverage once it is included in the scanning range.

In helical scanning, it is usually difficult to locate the start and the end of scanning range necessary to image the temporal bone. Additional scanning range is also necessary to facilitate multiplanar reformation. As a result, the scanning range is generally larger (approximately $50 \mathrm{~mm}$ ) than that for sequential imaging in clinical practice. Furthermore, in sequential scanning, gantry angulation results in a decrease in $\mathrm{CTDI}_{\mathrm{vol}}$ to some degree, but this is unavailable in helical scanning. The effective dose $(0.803 \mathrm{mSv})$ of temporal bone CT in the helical scanning mode is higher than the doses of direct axial $(0.64$ $\mathrm{mSv})$ and coronal scanning $(0.57 \mathrm{mSv})$ alone but lower than their combined effective doses. The 2 direct scanning modes are usually performed consecutively in a single examination, leading to a gross effective dose of $1.21 \mathrm{mSv}$ and a lens dose of $52.69 \mathrm{mGy}$. These doses reflect an increase relative to those in the helical mode of 1.51 and 1.31 times, respectively. A modified helical mode obtained in a plane that will avoid direct radiation to the lens can reduce the lens organ dose by $74.3 \%$. We recommend that the modified helical scanning mode be used to image the temporal bone in clinical practice.

There are 2 main limitations to this study. First, the exsomatized cadaveric heads were stored in a formalin solution for a long period. The muscular tissues and encephalic structures dehydrated noticeably during this time. As a result, there was decreased $x$-ray attenuation in the cadaveric heads, and the radiation dose measured with TLDs was likely higher than that which would be measured in a living human. Second, the Brilliance 64-section CT scanner used in this study displays mAs section (ie, effective mAs) equal to the mAs of 1 tube rotation divided by pitch. In this study, we required that the CTDI ${ }_{\mathrm{vol}}$ of each helical scan be equivalent to that of the sequential scan to compare the 2 lens dose values; however, the real tube output is $220 \mathrm{mAs}$ in the sequential scanning mode and $69.3 \mathrm{mAs}$ in the helical scanning mode. We did not optimize the dose of the sequential scan to find a fitting mAs.

\section{References}

1. McCollough C, Cody, D, Edyvean S, et al. The measurement, reporting, and management of radiation dose in CT. No. 96. Technical report. American Association of Physicists in Medicine. 2008. Available at: http://www.aapm.org/ pubs/reports/. Accessed March 20, 2009

2. ACR practice guideline for the performance of computed tomography (CT) of the extracranial head and neck in adults and children, revised 2006 (Res. 12,17,35). Available at: http://www.acr.org/SecondaryMainMenuCategories/ quality_safety/guidelines/nuc_med/fdg_pet_ct.aspx. Accessed November 25, 2008

3. Tweed JJ, Davies ML, Faulkner K, et al. Patient dose and associated risk due to radiological investigation of the internal auditory meatus. Br J Radiol 1991; 64:447-51

4. Siddle KJ, Sim LH, Case CC. Radiation doses to the lens of the eye during computerised tomography of the orbit: a comparison of four modern computerised tomography units. Australas Radiol 1990;34:323-25

5. Geleijns J, Van Unnik JG, Zoetelief J, et al. Comparison of two methods for assessing patient dose from computer tomography. Br J Radiol 1994;67: 360-65

6. Poletti JL. Patient doses from CT in New Zealand and a simple method for estimating effective dose. Br J Radiol 1996;69:432-36

7. Maclennan AC, Hadley DM. Radiation dose to the lens from computed tomog raphy scanning in a neuroradiology department. Br J Radiol 1995;68:19-22

8. Nishizawa K, Maruyama T, Takayama M, et al. Determinations of organ doses and effective dose equivalents from computed tomographic examination. $\mathrm{BrJ}$ Radiol 1991;64:20-28

9. Torizuka T, Hayakawa K, Satoh Y, et al. High-resolution CT of the temporal bone: a modified baseline. Radiology 1992;184:109-11

10. Funama Y, Awai K, Shimamura M, et al. Reduction of radiation dose at HRCT of the temporal bone in children. Radiat Med 2005;23:578-83

11. Lee CP, Black ML, Lamb RB, et al. CT evaluation of the temporal bone ossicles by using oblique reformations. AJNR Am J Neuroradiol 1989;10:431-33

12. Husstedt HW, Prokop M, Dietrich B, et al. Low-dose high-resolution CT of the petrous bone. J Neuroradiol 2001;27:87-92

13. Siebert E, Klingebiel R. Pediatric low-dose CT: skull base. Visions 2008;12: 62-65

14. Klingebiel R, Bauknecht HC, Kaschke O, et al. High-resolution petrous bone imaging using multi-slice computerized tomography. Acta Otolaryngol 2001; 121:632-36

15. Vazquez E, Castellote A, Piqueras J, et al. Imaging of complications of acute mastoiditis in children. Radiographics 2003;23:359-72

16. Hopper KD, Neuman JD, King SH, et al. Radioprotection to the eye during CT scanning. AJNR Am J Neuroradiol 2001;22:1194-98

17. Caldemeyer KS, Sandrasegaran K, Shinaver CN, et al. Temporal bone: comparison of isotropic helical CT and conventional direct axial and coronal CT. AJR Am J Roentgenol 1999;172:1675-82

18. Lutz J, Jäger V, Hempel MJ, et al. Delineation of temporal bone anatomy: feasibility of low-dose 64-row CT in regard to image quality. Eur Radiol 2007; 17:2638-45. Epub 2007 Mar 7

19. International Commission on Radiological Protection. ICRP Publication 103: Recommendations of the ICRP: Annals of the ICRP. Vol 21. Oxford, UK: Perga 